Open Access

\title{
Correction to: Multimodal treatment of pediatric patients with Askin's tumors: our experience
}

Silvia Triarico ${ }^{1 *}$, Giorgio Attinà ${ }^{1}$, Palma Maurizi ${ }^{1}$, Stefano Mastrangelo ${ }^{1}$, Lorenzo Nanni ${ }^{2}$, Vito Briganti ${ }^{3}$, Elisa Meacci ${ }^{4}$, Stefano Margaritora ${ }^{4}$, Mario Balducci ${ }^{5}$ and Antonio Ruggiero ${ }^{1}$

\section{Correction}

In the original article mentioned above, the name of the sixth author was wrongly mentioned as "Vincenzo Briganti" instead of "Vito Briganti".

The original article has been corrected.

\begin{abstract}
Author details
'Paediatric Oncology Unit, A. Gemelli University Hospital, Catholic University of Sacred Hearth, Largo A. Gemelli, 8, 00168 Rome, Italy. ${ }^{2}$ Pediatric Surgery, Unit Gemelli University Hospital, Catholic University of Sacred Heart, Rome, Italy. ${ }^{3}$ Pediatric Surgery Unit, San Camillo Forlanini Hospital, Rome, Italy. ${ }^{4}$ Thoracic Surgery Unit, Gemelli University Hospital, Catholic University of Sacred Heart, Rome, Italy. ${ }^{5}$ Radiotherapy Unit, Gemelli University Hospital, Catholic University of Sacred Heart, Rome, Italy.
\end{abstract}

Published online: 25 September 2018

\section{Reference}

1. Triarico S, Attinà G, Maurizi P, Mastrangelo S, Nanni L, Briganti V, Meacci E, Margaritora S, Balducci M, Ruggiero A. Multimodal treatment of pediatric patients with Askin's tumors: our experience. World J Surg Oncol. 2018;16: 140. https://doi.org/10.1186/s12957-018-1434-2.

\footnotetext{
* Correspondence: silviatriarico@libero.it

'Paediatric Oncology Unit, A. Gemelli University Hospital, Catholic University

of Sacred Hearth, Largo A. Gemelli, 8, 00168 Rome, Italy
} 